\title{
Acolhimento hospitalar em tempos de pandemia de COVID-19: relato de experiência
}

\author{
Hospital care in times of COVID-19 pandemic: experience report
}

\section{Atención hospitalaria en tiempos de la pandemia de COVID-19: informe de experiencia}

Danielle Cortêz da Silva ${ }^{\text {* }}$, Bruna Regina dos Santos ${ }^{2,3}$, Kemily Kawana Oliveira da Silva²,3, Bárbara Vitória Cesário da Silva ${ }^{2,3}$, Desirée Ariane Modos Figueira1', Magali Godoy Pereira Cardoso", Elisana Agatha lakmiu Camargo Cabulon ${ }^{1}$, Patrícia Aroni', Raquel Gvozd Costa ${ }^{1}$.

\section{RESUMO}

Objetivo: Descrever a experiência de uma instituição de alta complexidade no acolhimento das famílias e dos pacientes hospitalizados durante a pandemia de COVID-19. Relato de experiência: Estudo qualitativo descritivo, do tipo relato de experiência, acerca do acolhimento hospitalar na pandemia de COVID-19 em um hospital universitário de alta complexidade do norte do Paraná. O estudo foi realizado por uma comissão de acolhimento multidisciplinar entre junho e novembro de 2020 e discutido em duas categorias, sendo a primeira "Proposta Articulada de Acolhimento", e, a segunda, "O que aprendi acolhendo você e a sua família". Considerações finais: A experiência fortaleceu o vínculo entre profissional, família e paciente, na forma como eles se constroem e a na potência de afetar a vida. A iniciativa promoveu o acolhimento do indivíduo e de seus familiares, propiciando, assim, uma experiência mais afetiva durante o período de hospitalização do paciente na pandemia. Espera-se que essa experiência sirva de exemplo para outras instituições implementarem ações de acolhimento durante e após a pandemia de COVID-19.

Palavras-chave: Acolhimento, Infecções por coronavírus, Humanização da assistência, Equipe de assistência ao paciente.

\section{ABSTRACT}

Objective: To describe the experience of a highly complex institution in welcoming families and hospitalized patients during the COVID-19 pandemic. Experience report: Qualitative descriptive study of the experience report type about hospital care in the COVID-19 pandemic in a high complexity university hospital in northern Paraná. The study was carried out by a multidisciplinary welcoming committee between June and November 2020 and discussed in two categories, the first being "Proposed Articulated Reception" and the second "What I learned welcoming you and your family". Final considerations: The experience strengthened the bond between professional, family and patient, in the way they are built and in their power to affect life. The initiative promoted the welcoming of individuals and their families, thus providing a more affective experience during the period of hospitalization of the patient in the COVID-19 pandemic. Hopes that this experience will serve as an example for other institutions to implement actions of support during and after the COVID -19 pandemic.

Key words: Reception, Coronavirus infections, Humanization of care, Patient care team.

\section{RESUMEN}

Objetivo: Describir la experiencia de una institución de alta complejidad en la acogida de familias y pacientes hospitalizados durante la pandemia de COVID-19. Informe de experiencia: Estudio descriptivo cualitativo del tipo de informe de experiencia sobre la atención hospitalaria en la pandemia de COVID-19 en un hospital universitario de alta complejidad. en el norte de Paraná. El estudio fue realizado por un comité de acogida multidisciplinario entre junio y noviembre de 2020 y se debatió en dos categorías, la primera de "Recepción articulada propuesta" y la segunda "Lo que aprendí dándote la bienvenida a ti y a tu familia". Consideraciones finales: La experiencia fortaleció el vínculo entre el profesional, la familia y el paciente, en la forma en que se construyen y en su poder de incidir en la vida. La iniciativa promovió la recepción del individuo y sus familias, brindando así una experiencia más afectiva durante el período de internación del paciente en la pandemia COVID-19. Espera que esta experiencia sirva de ejemplo para que otras instituciones implementen acciones de apoyo durante y después de la pandemia de COVID -19.

Palabras clave: Recepción, Infecciones por coronavirus, Humanización de la atención, Equipo de atención al paciente.

1 Universidade Estadual de Londrina (UEL), Londrina - PR. *E-mail: danielle16cortez@gmail.com

2 Faculdade Pitágoras, Londrina - PR.

${ }^{3}$ Programa "O Brasil Conta Comigo", Governo Federal, Brasil.

SUBMETIDO EM: 12/2021 | ACEITO EM: 12/2021 | PUBLICADO EM: 1/2022 


\section{INTRODUÇÃO}

O acolhimento, em suas inúmeras dimensões, define-se por aceitar, dar crédito, admitir, agasalhar, atender e receber, representando uma ação de aproximação, ou seja, uma atitude de inclusão (FERREIRA $A B H, 2005)$. No Brasil, tendo em vista garantir os princípios do Sistema Único de Saúde (SUS), o acolhimento é uma diretriz de importância política, ética e estética da Política Nacional de Humanização (PNH). Implantada em 2003, a PNH se caracteriza por ser uma estratégia de encontro e de construção de vínculo em busca de acesso à saúde com resolutividade, qualidade e assertividade dentro do SUS, a qual inclui gestores, trabalhadores e usuários, agregando suas diferenças para potencializar as transformações na maneira de cuidar e de implementar processos de trabalho (MINISTÉRIO DA SAÚDE, 2020b).

Especialmente durante o processo de hospitalização, o acolhimento é um dos dispositivos essenciais para a garantia do bem-estar e do protagonismo do paciente, visto que, nessas condições, o indivíduo se encontra privado de suas atividades cotidianas e do conforto do lar e acaba sendo submetido a rotinas restritivas, impostas por pessoas desconhecidas. Tudo isso pode causar desconforto, angústia, medo e até mesmo dores, tornando o processo ainda mais vulnerável a outros agravos de saúde (VITÓRIA AL e ASSIS CL, 2015; GALÁN GONZÁLEZ-SERNA JM, et al., 2017; NEVES L, et al., 2018).

A presença de um familiar ou de um amigo, seja como acompanhante ou como visitante, pode proporcionar conforto e reforçar a segurança para o paciente. Todavia, além das fragilidades vivenciadas pelos usuários no decorrer da hospitalização, a família também se encontra em condições de vulnerabilidade por passar por tais situações com seu ente. Nesse contexto, estar junto com a familia e com o paciente, isto é, acolhê-los, acaba sendo uma postura ética que implica a escuta e o reconhecimento do protagonismo do usuário, pois o cuidado, quando centrado na família e nas necessidades biopsicossociais do indivíduo, melhora o esgotamento e o sof rimento mental de todos os envolvidos (HART JL, et al., 2020; MENEGUIN S, et al., 2019; VITÓRIA AL e ASSIS CL, 2015).

Além disso, estar junto à família e ao paciente faz parte das reações humanas, do reconhecimento do outro, das suas diferenças, das suas dores, das suas alegrias e dos seus modos de viver, contribuindo para a construção da sua própria humanidade. Acolher está presente em todas as relações humanas e é um compromisso com as necessidades de saúde de todos os indivíduos (ALVES ATLS, et al., 2017).

Atualmente, uma nova realidade tem sido experienciada pelos serviços de saúde, que foram surpreendidos pela pandemia causada pelo Coronavírus (COVID-19), um vírus altamente transmissível, que afeta principalmente o sistema respiratório. Em decorrência disso, rapidamente foram tomadas decisões que demandaram significativas mudanças na rotina institucional, o que afetou diretamente os protocolos de humanização e o acolhimento, levando em conta a segurança coletiva (MINISTÉRIO DA SAÚDE, 2020c; AGUIAR NM, et al., 2021).

No ambiente hospitalar, foram necessárias diversas adequações relacionadas à estrutura e aos processos, bem como houve limitação quanto aos fluxos interno e externo de pessoas, a fim de reduzir sua transição e o risco de infecção, sendo preciso restringir a permanência de acompanhantes e de visitantes junto aos pacientes hospitalizados, tornando o acolhimento um novo desafio (HART JL, et al., 2020).

As condições impostas pela pandemia de COVID-19 exigiram das equipes a elaboração de novas ações para assegurar o vínculo entre usuário, família e colaboradores. Nesse sentido, procuraram-se novas maneiras de acolhimento e viabilizar a presença da família de modo não físico (CRISPIM D, et al., 2020).

Diante disso, este estudo objetivou descrever a experiência de uma instituição de alta complexidade no acolhimento das famílias e dos pacientes hospitalizados durante a pandemia de COVID - 19.

\section{RELATO DA EXPERIÊNCIA}

Estudo qualitativo descritivo, do tipo relato de experiência, acerca do acolhimento hospitalar na pandemia de COVID-19 em um hospital universitário de alta complexidade do norte do Paraná. A instituição é composta por 467 leitos, divididos entre leitos de Internação e leitos de Unidade de Terapia Intensiva (UTI), que atendem usuários do SUS em todos os ciclos de vida. 
Com o início da pandemia de COVID -19 e o alto risco de transmissão do vírus, não foi possível manter as rotinas dos acompanhantes e de visita presencial aos pacientes internados na instituição. Contudo, considerando o grau de sof rimento de todos os envolvidos, desde os líderes profissionais até os pacientes e seus familiares, o hospital criou uma Comissão de Acolhimento e Comunicação (CCA), com o intuito de assegurar a comunicação entre os pacientes, os familiares e os profissionais de saúde.

A comissão foi constituída por uma equipe multidisciplinar, que envolveu enfermeiros, médicos, psicólogos, assistentes sociais e graduandos de enfermagem cedidos pelo programa do Governo Federal "Brasil Conta Comigo". Os autores atuam como membro da comissão, realizando toda a articulação, a busca ativa e o contato com a família e com o paciente.

Este estudo relata a experiência vivenciada entre junho e novembro de 2020. Para melhor compreensão da experiência, optou-se por descrevê-la em duas categorias, sendo a primeira "Proposta Articulada de Acolhimento", e, a segunda, "O que aprendi acolhendo você e a sua família".

\section{Categoria 1: proposta articulada de acolhimento}

Por tratar-se de um hospital de alta complexidade, a instituição atende cerca de 93 municípios da região. É possível identificar diferentes realidades sociais, como pacientes que dispõem de tecnologias para comunicação e pacientes que não possuem nenhum tipo de meio de comunicação, como telefone celular ou residencial. Visando atender a esses contrastes, criou-se um fluxo de comunicação da CCA, com o propósito de garantir a informação segura a todos os envolvidos (Figura 1).

Figura 1 - Fluxo de Comunicação da CCA.

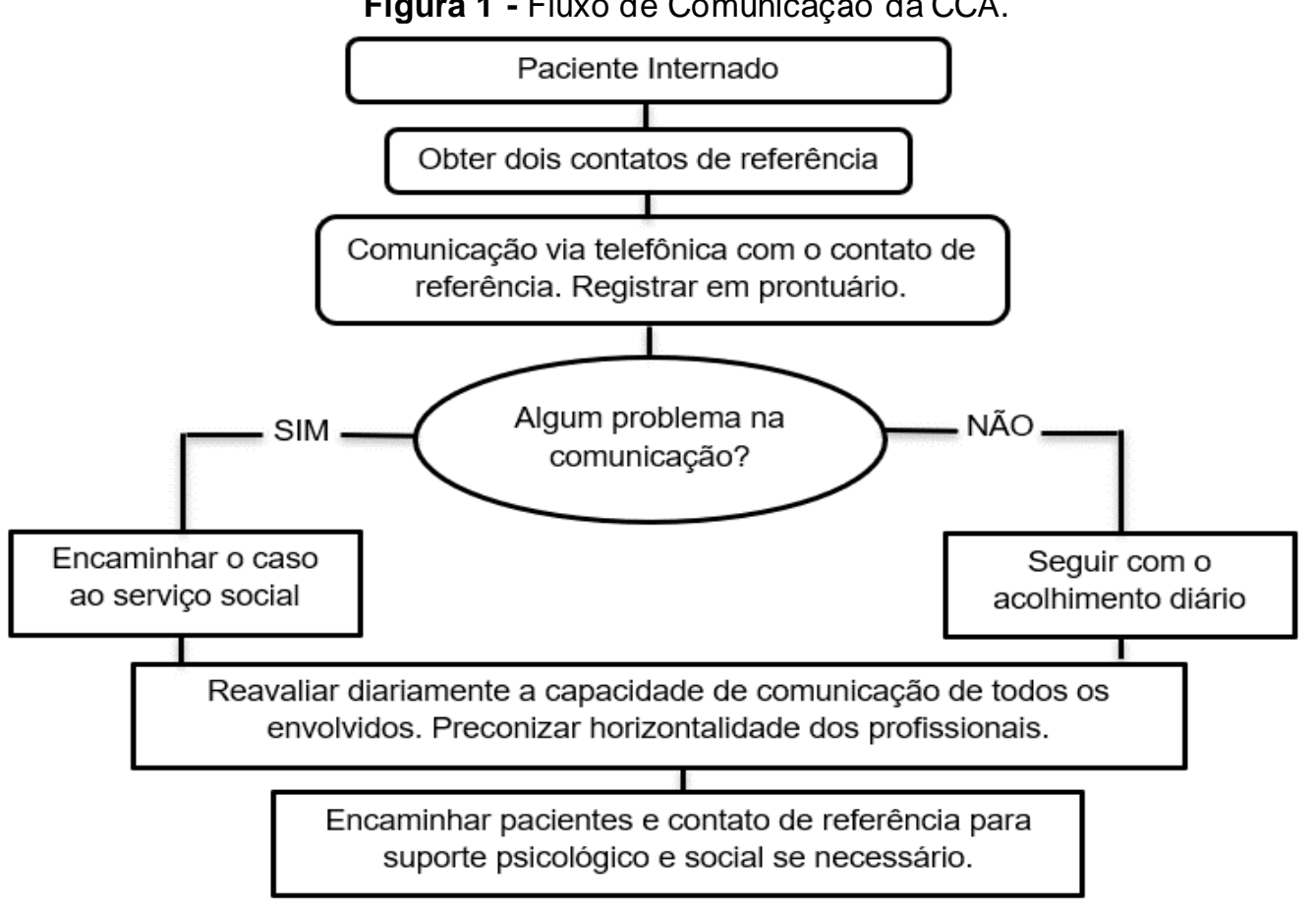

Nota: Imagem construída pelo Microsoft Word. Fonte: Silva DC, et al., 2021.

Na primeira parte do fluxo, pretendeu-se identificar os pacientes internados, dando prioridade para aqueles que não conseguiam contato com família e com os amigos. A partir daí, definia-se junto ao paciente dois contatos de ref erência, com familiar ou com amigo, para que as informações sobre o caso fossem repassadas. A comunicação era feita via telefone, com o contato estabelecido uma vez ao dia e registrada em prontuário.

A concepção do fluxo buscou identificar as dificuldades de comunicação de cada caso e uma resolução integrada e multiprofissional da situação. Em circunstâncias de dificuldade, a equipe realizava integração junto às Unidades Básicas de Saúde (UBS) e às Secretárias de Saúde do Município de residência do paciente. Se necessário, os casos recebiam suporte social e psicológico da equipe multiprofissional. 
Para permear todo o atendimento estipulado pelo fluxo da CCA, desenvolveu-se um instrumento guia, com o intento de direcionar o processo de comunicação entre a equipe de saúde, o contato de referência e o paciente. $O$ instrumento foi formado em três etapas: I Contatos de referência, II Situação de saúde e necessidades do paciente, e III Acolhimento e necessidades da família.

Figura 2 - Instrumento guia para visita hospitalar.

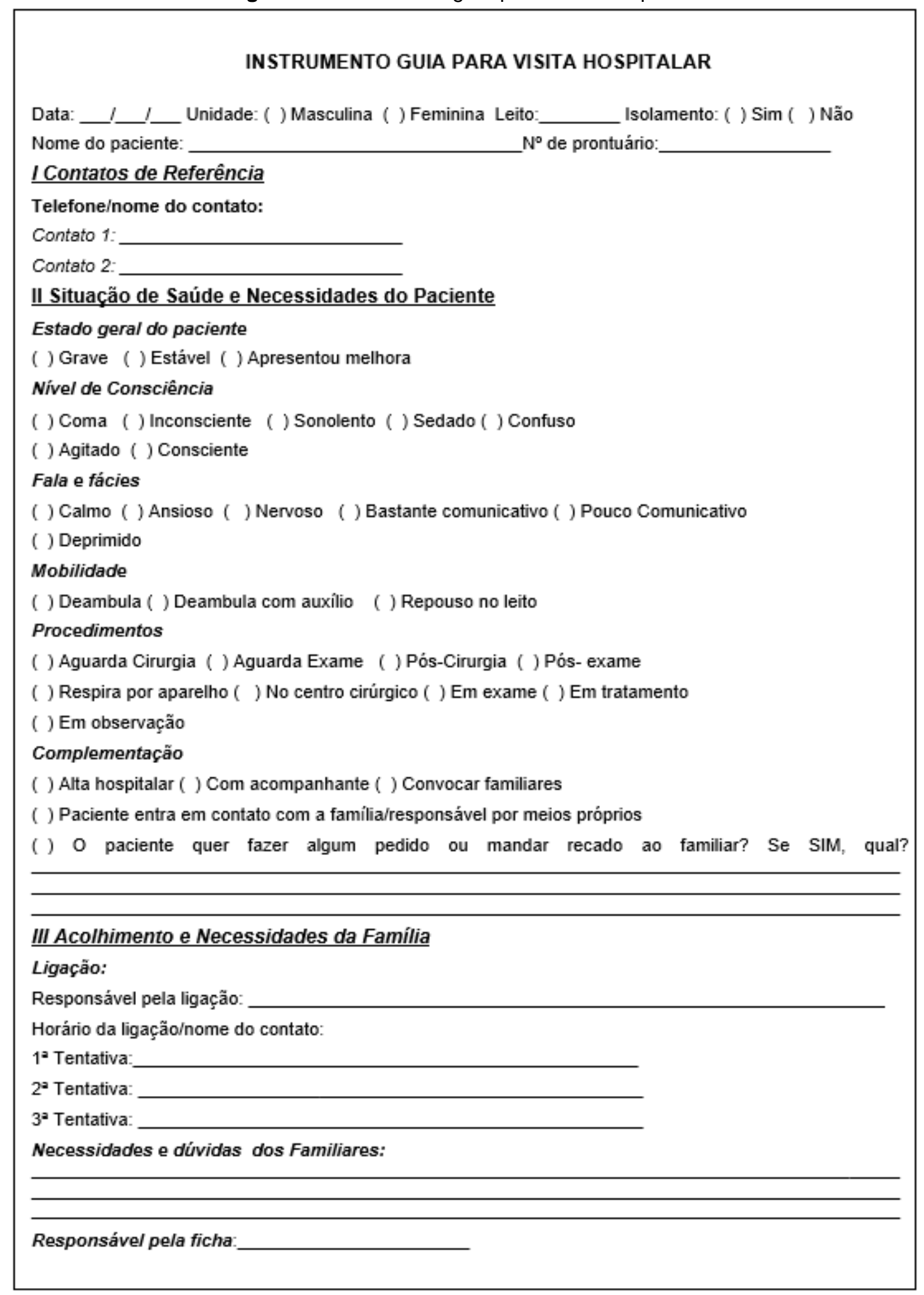

Nota: Imagem construída pelo Microsoft Word. Fonte: Silva DC, et al., 2021. 
As ligações eram realizadas diariamente pelos estudantes do programa "Brasil Conta Comigo" (BCC), sendo que a primeira parte do instrumento teve o objetivo de nortear o processo de comunicação dos estudantes junto aos pacientes. Nessa sessão, a interação com o paciente tinha como finalidade eleger dois contatos de sua preferência, seja familiar ou amigo, como referência. O paciente, desde que em condições clínicas, tinha total autonomia para decidir quem seriam tais contatos.

A segunda parte do instrumento foi direcionada às necessidades biopsicossociais do paciente, em que os estagiários do programa BCC conduziam uma abordagem segundo suas necessidades básicas, identificando seu estado de saúde. De fato, essa etapa teve como intuito compilar informações sobre a condição geral do paciente, e não fornecer boletins médicos, o que é atribuição exclusivamente da categoria médica.

Em face da exigência de um parecer médico identificado pelo profissional ou solicitado pelo contato de referência, um encontro presencial ou remoto era agendado por intermédio do serviço social. Na escolha do tipo de encontro com a equipe médica foram respeitad as as limitações e as necessidades do paciente e do contato de referência e as questões de vulnerabilidade impostas pela pandemia. Sendo assim, aconteciam tanto encontros remotos como presenciais entre a equipe de saúde e o contato de referência do paciente.

A última parte do instrumento referiu-se ao contato com os familiares ou com os amigos por meio telefônico. Os estagiários do BCC procediam a visita seguindo o instrumento guia, procurando criar vínculo com o paciente e com o contato de referência, ao qual eram repassadas informações gerais acerca da situação do paciente. Ademais, a abordagem ainda permitia que recados fossem enviados entre o contato de referência e o paciente.

Na prática, foi possível notar que os envolvidos aderiram à iniciativa, uma vez que os pacientes frequentemente solicitavam objetos pessoais ou mesmo enviavam mensagem de af eto aos seus contatos de referência. Prontamente, os pedidos eram atendidos e as demandas eram viabilizadas pela família, em parceria com a instituição. Todo o processo de solicitação e de entrega dos pedidos ficava sob responsabilidade do estagiário do BCC, dada a conexão que ele construía com o contato de referência e com o paciente por meio das ligações.

As incertezas concernentes ao novo vírus circulante, SARS-CoV-2, e à Covid-19 foram motivo de mobilização de pessoas mundialmente, com a ambição de organizar respostas partindo das diferentes realidades sociais e sanitárias e dos sistemas de saúde envolvidos. Foi nesse cenário, com base nos princípios de comunicação efetiva, de acolhimento familiar, de respeito e de direito à informação, que se estruturou a CCA.

\section{Categoria 2: 0 que aprendi acolhendo você e a sua família}

A CCA foi instituída em meio a uma conjuntura de dúvidas e de euforia quanto a um novo vírus circulante e potencialmente fatal. Foi preciso aprender a lidar com a doença tanto no campo pessoal como no profissional e a trabalhar com o turbilhão de sentimentos causados pelo desconhecido.

Durante a realização da visita ao paciente, nas conversas com os profissionais dos setores e nas ligações com os contatos de referência, a ansiedade era constante, o que estava atrelado ao risco de contágio, ao medo da morte sem despedida e às incertezas ocasionadas pelo processo de hospitalização.

A cada ligação e visita ao paciente, foi necessário ressignificar o modo de acolher, distinguindo as próprias emoções e buscando estratégias para trabalhar com elas. As capacidades de ouvir atentamente, de saber falar e de ficar em silêncio foram aprimoradas, na impossibilidade de um abraço ou de um aperto de mão. Praticar acolhimento em tempos de pandemia ultrapassou a barreira da transmissão de informações por técnicas postas e fez de um momento de vulnerabilidade um aprendizado mútuo.

Compaixão em forma de atitude foi a sensação que permeou todos os atendimentos. A cada ligação feita ou recebida foi essencial ouvir com paciência e sensibilidade, respeitando a singularidade de cada paciente e de cada contato de ref erência. O acolhimento foi sentido e vivido, foi como cuidar de si mesmo ou da própria família. 
Considerar os aspectos subjetivos, culturais e individuais de cada paciente oportunizou sentir junto e ser tocado pela fragilidade do outro, mas também por aquilo que trazia conforto e esperança. Acolher os pacientes e suas famílias trouxe a compreensão de que, apesar de lidarmos com a doença e com o sofrimento que a permeia, temos que manter o entusiasmo e que valorizar cada momento.

Muitas foram as angústias da família e do paciente, relativas tanto à doença quanto ao extinto de cuidar do ser humano. Muitos dos questionamentos dos familiares eram: "Ele dormiu bem a noite?" ou "Ele comeu bem?"; já os pedidos comuns eram: "Posso enviar meias? Ele não dorme bem sem meias nos pés!". A experiência da CCA proporcionou dedicação af etiva e empática de não somente se sentir no lugar do outro, mas de ser motivado a agir em prol de aliviar preocupações e cargas de sentimentos que fragilizam o outro.

Fazer parte da CCA mostrou que sentir é estar suscetível, mas que esse processo é fundamental para ir além e tornar-se um profissional capaz de construir uma relação mais humanizada. Acolher aproxima a essência de ser humano e evidencia que, por maior que seja a dor, estar junto ao paciente e à família traz alívio e fortalece a equipe, tornando o processo de hospitalização mais tenaz.

\section{DISCUSSÃO}

Hoje, vive-se em um mundo globalizado, altamente tecnológico e informatizado, em que a nanotecnologia se faz presente em diversos campos da área da saúde. Nesse quadro, o uso dessas tecnologias durante o atendimento em saúde possui um caráter biológico-médico-hegemônico, no qual o foco é a queixa do paciente, tornando o acolhimento em saúde e a humanização grandes desafios (SILVA RMCRA, et al., 2015; QUADROS LCT, et al., 2020; SOUSA KHJF, et al., 2019; RIBEIRO ALTS, et al., 2020).

Contudo, a pandemia de COVID-19 utilizou dessa tecnologia, que até então era vista como a vilã que afastava e fragilizava o acolhimento em saúde, como uma estratégia de aproximação entre profissionais, pacientes e famílias. O cenário imposto alcançou feitos inimagináveis, porém se vive em um país de contrastes e de desigualdades sociais, em que é preciso, por muitas vezes, produzir saúde com escassez de recursos (MINISTÉRIO DA SAÚDE, 2020a; QUADROS LCT, et al., 2020).

O acolhimento é uma tecnologia leve, mas de impacto e de resultados importantes na atenção à saúde, todavia classificado como uma ação não relevante por muitos profissionais. Prevenir, cuidar, proteger, tratar, recuperar e promover saúde são alguns dos desafios enfrentados no ambiente de trabalho. Muitas vezes, o profissional "adormece" a escuta, causando indiferença no tocante à necessidade do outro. Isso produz uma falsa sensação de proteção dos sentimentos, entorpecendo a sensibilidade e enfraquecendo os laços entre paciente e profissional, laços estes substanciais para o acolhimento em saúde. Neste estudo, durante 0 atendimento dos pacientes e dos familiares, foi imprescindível ultrapassar essas barreiras pessoais de proteção e reativar a capacidade de ouvir atentamente, na medida em que a pandemia exigiu a proximidade por meio da distância, dentro e fora do ambiente hospitalar (MINISTÉRIO DA SAÚDE, 2010a; QUADROS LCT, et al., 2020).

Nesse contexto inusitado, a articulação estratégica da gestão com propostas na mudança do processo de trabalho foi primordial, representando o compromisso com os usuários do serviço de saúde e com os trabalhadores, além de um recurso para a humanização em saúde (MINISTÉRIO DA SAÚDE, 2010b; SILVA JÚNIOR, et al., 2020; QUADROS LCT, et al., 2020).

A experiência vivenciada neste estudo valorizou o vínculo entre profissional, famíliae paciente, na maneira como eles se constroem e na potência de af etar a vida. A iniciativa proporcionou o acolhimento do indivíduo e de seus familiares, propiciando, assim, uma vivência mais af etiva durante o período de hospitalização do paciente na pandemia COVID-19. Ainda, contribuiu para acalentar os familiares, angustiados tanto pelas incertezas acerca do quadro clínico quanto pela privação do convívio com seus entes queridos. Portanto, a fim de aprimorar essa experiência, pretende-se implementar a visita virtual, por meio da qual os estagiários farão o contato com a família via chamada de vídeo, ampliando, pois, a comunicação e o contato visual de modo seguro para todos. 


\section{REFERÊNCIAS}

1. ALVES ATLS, et al. Implantação de um boletim informativo como proposta de humanização no pronto-socorro de um hospital público. Revista Baiana Saúde Pública, 2017;41(2): 537-550.

2. AGUIAR NM, et al. Projeto aplicativo e a humanização no SUS: a experiência de acadêmicos de medicina. CuidArte. Enfermagem, 2021;15(1):61-66.

3. CRISPIM DH, et al. Comunicação difícil e Covid-19: recomendações práticas para comunicação e acolhimento em diferentes cenários da pandemia. 2020. Disponível em: https://ammg.org.br/wpcontent/uploads/comunica\%C3\%A7\%C3\%A3o-COVID-19.pdf.pdf. Acessado em: 17 de abril de 2021.

4. FERREIRA ABH. Dicionário Aurélio básico da língua portuguesa. Rio de Janeiro: Nova Fronteira, 2005.

5. GALÁN GONZÁLEZ-SERNA JM, et al. Development and validation of the Hospitality Axiological Scale for Humanization of Nursing Care. Revista Latino-Americana de Enfermagem, 2017;25:e2919.

6. HART JL, et al. Family-centered care during the COVID-19 era. Journal of Pain and Symptom Management, 2020; 60(2): e93-e97.

7. LETÍCIA A. Vivências e estratégias de enfrentamento em acompanhantes de familiar hospitalizado em uma unidade hospitalar do município de Cacoal-RO. Aletheia, 2015;46: 16-33.

8. MENEGUIN S, et al. O significado de conforto na perspectiva de familiares de pacientes internados em UTI. Nursing, 2019;22(252):2882-2886.

9. MINISTÉRIO DA SAÚDE. Saúde mental e atenção psicossocial na Covid-19:a quarentena na covid-19: orientações e estratégias de cuidado. 2020a. Disponível em: https://www.fiocruzbrasilia.fiocruz.br/wpcontent/uploads/2020/04/Sa\%c3\%bade-Mental-e-Aten\%c3\%a7\%c3\%a3o-Psicossocial-na-Pandemia-Covid-19-Aquarentena-na-Covid-19-orienta\%c3\%a7\%c3\%b5es-e-estrat\%c3\%a9gias-de-cuidado.pdf. Acessado em: 15 de abril de 2021.

10. MINISTÉRIO DA SAÚDE. Saúde mental e atenção psicossocial na Covid-19: orientações às/aos psicólogas/os hospitalares. 2020b. Disponível em: https://www.fiocruzbrasilia.fiocruz.br/wpcontent/uploads/2020/04/cartilha_psicologos_hospitalares.pdf. Acessado em: 15 de abril de 2021.

11. MINISTÉRIO DA SAÚDE. Saúde mental e atenção psicossocial na Covid-19: processo de luto na Covid19. 2020c. Disponível em: http://renastonline.ensp.fiocruz.br/sites/default/files/arquivos/recursos/saude-mental-e-atencaopsicossocial-na-pandemia-covid-19-processo-de-luto-no-contexto-da-covid-19.pdf. Acessado em: 15 de abril de 2021.

12. MINISTÉRIO DA SAÚDE. Saúde mental e atenção psicossocial na Covid-19: recomendações aos psicólogos para o atendimento online. 2020d. Disponível em: https://www.fiocruzbrasilia.fiocruz.br/wpcontent/uploads/2020/04/Sa\%c3\%bade-e-Mental-e-Aten\%c3\%a7\%c3\%a3o-Psicossocial-na-Pandemia-Covid-19recomenda\%c3\%a7\%c3\%b5es-aos-psic\%c3\%b3logos-para-o-atendimento-online-1.pdf. Acessado em: 15 de abril de 2021.

13. MINISTÉRIO DA SAÚDE. Acolhimento nas práticas de produção de saúde.2. ed. Brasília, DF: Editora do Ministério da Saúde, 2010a; 44p. Disponível em: https://bvsms.saude.gov.br/bvs/publicacoes/acolhimento_praticas_producao_saude.pdf. Acesso em: 27 Junho de 2021.

14. MINISTÉRIO DA SAÚDE. Humaniza SUS: documento base para gestores e trabalhadores do SUS. $4^{\stackrel{a}{a}}$ ed. Brasília, DF: Editora do Ministério da Saúde, 2010b. Disponível em: https://bvsms.saude.gov.br/bvs/publicacoes/humanizasus_documento_gestores_trabalhadores_sus.pdf. Acessado em: 10 de Junho de 2021.

15. NEVES $L$, et al. The impact of the hospitalization process on the caregiver of a chronic critical patient hospitalized in a Semi-Intensive Care Unit. Escola Anna Nery, 2018;22(2): 1-8.

16. QUADROS LCT, et al. Acolhimento psicológico e afeto em tempos de pandemia: práticas políticas de afirmação da vida. Psicologia \& Sociedade, 2020;32: e020016.

17. RIBEIRO ALTS, et al. Dispositivos e contribuições da Política Nacional de Educação Permanente e m Saúde e Política Nacional de Humanização: para o fortalecimento dos processos de trabalho de gestores e profissionais da atenção primária no Tocantins. Palmas: Secretaria de Estado da Saúde, 2020; 38 p.

18. SALVATI CO, et al. Humanization of the hospital:participatory construction of knowledge and practices on care and ambience. Revista da Escola de Enfermagem da USP, 2021;55: e20200058.

19. SILVA JÚNIOR JNB, et al. Behavior of nursing professionals and the implementation of hospital humanization policy. Revista de Pesquisa Cuidado é Fundamental Online, 2020;12:471-478.

20. SILVA RMCRA, et al. A produção discursiva dos profissionais acerca da humanização e saúde: singularidade, direito e ética. Revista Latino-Americana de Enfermagem, 2015;23(5):936-944.

21. SONIS JD, et al. Humanism in the age of Covid-19: renewing focus on communication and compassion. Westem Journal of Emergency Medicine, 2020;21(3):499-502.

22. SOUSA KHJF, et al. Humanização nos serviços de urgência e emergência: contribuições para o cuidado de enfermagem. Revista Gaúcha de Enfermagem, 2019;40: e20180263.

23. VITORIA AL, ASSIS CL. Vivências e estratégias de enfrentamento em acom panhantes de familiar hospitalizado em uma unidade hospitalar do município de Cacoal-RO. Aletheia [online], 2015; (46): 16-33. 